\title{
Determinismo, no linealidad y caos Comentarios y reflexiones sobre la obra "Caos y Orden"
}

\author{
Mariano Matilla Garcia \\ Dpto. Economía Aplicada Cuantitativa. UNED \\ Francisco José Vázouez Hernández
}

Dpto. Análisis Económico: Economía Cuantitativa. Universidad de Madrid

El libro «Caos y Orden», escrito por el profesor Antonio Escohotado y galardonado con el premio Espasa de Ensayo 1999, constituye un magnífico ejemplo de lectura que incita a la reflexión. Desde este compromiso. el autor nos adentra en la moderna teoría del caos y en sus proyecciones multidisciplinares, en un recorrido que va de áreas puramente científicas, como la Física y la Matemática, hasta terrenos humanísticos, como la Filosofía, la Historia y el Derecho, pasando por las denominadas Ciencias Sociales (Economía, Sociología y Política). Naturalmente, semejante empresa conlleva algunas limitaciones. Quizá, la más relevante, al menos desde nuestro punto de vista, es la insuficiente profundidad teórica con la que se abordan los principales conceptos de la teoría del caos. Esto motiva que ciertos términos, en concreto los relativos a determinismo, no linealidad y caos, como el propio concepto de atractor extraño, se empleen con poca precisión, propiciando en ocasiones, sobre todo en el lector inexperto en estos temas, una inadecuada comprensión de los mismos.

Nuestro propósito consiste, por una parte, en aclarar algunos de estos términos utilizados a lo largo del libro con escasa precisión, de ahí que a veces sea necesario apelar a la Matemática para dotar de suficiente rigor la exposición, y contribuir con ello a una mejor comprensión del tipo de paradigma en el que se circunscribe la teoría del caos. Por otra parte, esto permite revisar de forma sustancial las conclusiones derivadas de la lectura del texto, así como la aplicabilidad de este paradigma al dominio político e histórico. En este sentido, y como punto de partida, comenzamos con la noción de sistema dinámico.

La modelización teórica de un fenómeno real que se desarrolla a lo largo del tiempo, proviene de un ejercicio de abstracción y simplificación de la 
realidad ', en el que se extraen las variables (de estado) consideradas relevantes y se idealizan, por medio de una ley, la pautas que rigen sus cambios. Si el modelo teórico pretende endógena e históricamente ofrecer una explicación de la realidad, entonces los valores del estado presente deben ser consecuencia directa de estados anteriores. Así, un sistema dinámico puede ser entendido como la representación matemática de un proceso que cambia en el tiempo atendiendo a una cierta ley, que determina la evolución temporal del estado del sistema en función de su historia previa. En particular, un sistema dinámico discreto viene dado por una fórmula de recurrencia

$$
x_{t}=f\left(x_{t-1}\right)
$$

donde $t=1,2, \ldots$ representa el tiempo, medido a intervalos regulares y $x_{t}$ el estado del sistema en el instante (período) $t$, determinado por el estado en el instante anterior ${ }^{2,3}$. Así pues, el conocimiento del estado inicial $x_{0}$ basta para reconstruir toda la evolución del sistema, lo que confiere al sistema (modelo teórico) carácter determinista. En un sistema aleatorio o con incertidumbre, $x_{t}$ no se conoce exactamente a partir de $x_{t-1}$, en el mejor de los casos se conoce la probabilidad $p\left(z / x_{t-1}\right)$ de que $x_{t}$ tome un valor $z$ supuesto conocido $x_{t-1}$.

En este contexto, se denomina órbita de $x_{0}$ a la sucesión

$$
x_{0}, x_{j}=f\left(x_{0}\right), x_{2}=f\left(x_{1}\right)=f\left(f\left(x_{o}\right)\right)^{d e f}=f^{2}\left(x_{0}\right), \ldots \ldots \ldots, x_{t}=f^{f}\left(x_{0}\right), \ldots \ldots
$$

y se denota por $\gamma\left(x_{0}\right)$. Nótese que si la órbita (que, en general, es una sucesión infinita) $\gamma\left(x_{0}\right)$ consta de una cantidad finita de puntos, ésta se estabiliza bien en un punto fijo (por ejemplo, la órbita $x_{0}, x_{1}, x_{2}, x_{3}, x_{3}, x_{3}, x_{3}, \ldots$ se sitúa tras tres instantes en la órbita $\gamma\left(x_{3}\right)=\left\{x_{3}\right\}$ y $f\left(x_{3}\right)=x_{3}$ ) o bien en una órbita periódica $\left(x_{0}, x_{1}, x_{2}, x_{3}, x_{4}, x_{2}, x_{3}, x_{4}, \ldots\right.$ alcanza en dos instantes la órbita periódica $\gamma$ $\left(x_{2}\right)=\left\{x_{2}, x_{3}, x_{4}\right\}$ de período tres, siendo $f\left(x_{2}\right)=x_{3}, f\left(x_{3}\right)=x_{4}$, y $\left.f\left(x_{4}\right)=x_{2}\right)$.

Cuando la función $f$ que define el sistema es lineal, esto es, verifica $f(x)$ $=a x$ para algún número $a$ y para todo número $x$, siempre es posible obtener explícitamente la expresión de la órbita. Sin embargo, esto no sucede en general para sistemas no lineales; en esta situación se debe abandonar la idea de calcular su solución para pasar a afrontar la cuestión de cómo será su

${ }^{1}$ Resulta ilustrativo comparar esta idea con la sostenida en las páginas 116 y 117 , entre otras, de la obra en cuestión.

2 Para ser precisos, ésta es la expresión de un sistema dinámico discreto autónomo (independiente del tiempo), uni-dimensional (la variable de estado es escalar, dada por un único número en cada instante) y de primer orden (el estado sólo depende del anterior, podría suceder que el sistema tuviera más memoria y dependiera de varios estados anteriores). Se ha tomado esta referencia con el objeto de simplificar al máximo la exposición teórica.

${ }^{3}$ Por el mismo motivo, no se van a considerar los sistemas dinámicos continuos, que permiten representar procesos que cambian en cualquier instante ( $y$ no a intervalos regulares) y que vienen matemáticamente formulados por ecuaciones diferenciales. En cualquier caso, la mayor parte de los comentarios posteriores son extensibles al ámbito contínuo. 
comportamiento cualitativo futuro. Ahora bien, no conviene olvidar que las técnicas lineales, maltratadas en diversas partes del libro ${ }^{4}$, proporcionan un primer acercamiento a la dinámica del sistema; aunque la realidad sea inherentemente no lineal, el conocimiento del análisis lineal permite una aproximación local (cerca del punto que se esté considerando) a su comportamiento. Así pues, no es de extrañar el lógico desarrollo del pensamiento científico, y por ende del proceso educativo (sobre todo, desde el punto de vista pedagógico), que camina de la sencillez, caracterizada aquí por la linealidad, a la complejidad, propia de dinámicas no lineales. Que muchos fenómenos que habían sido considerados (fundamentalmente por simplicidad y operatividad) lineales ahora se planteen como no lineales (y así más cercanos a la realidad), no debería ser motivo de alegato contra la Ciencia (ni siquiera contra la que el autor califica de clásica), sino más bien de ensalzamiento de sus avances. De hecho, la dinámica no lineal ( $y$, en particular, la dinámica compleja) no suprime el análisis lineal, sino que lo utiliza como primera aproximación, para complementarlo finalmente con métodos más avanzados.

La moderna teoría de sistemas dinámicos, basada en análisis de tipo cualitativo, especialmente útil en modelos no lineales, tiene su origen en los trabajos de Poincaré de principios del siglo XX y fue desarrollándose por los estudios, entre otros, de Birkhoff, Levinson, Lyapunov, Smale y Kolmogorov. En este tipo de análisis, admitida la imposibilidad genérica de obtener la solución explícita de un sistema no lineal, lo que interesa es determinar el comportamiento asintótico (cuando el tiempo $t$ es suficientemente grande) de sus órbitas; es decir, suponiendo que las órbitas del sistema dinámico (1) permanecen acotadas, las preguntas ¿convergerán hacia un punto o hacia algún conjunto especial? y ¿partiendo de datos iniciales próximos, las órbitas correspondientes permanecerán relativamente próximas? apuntan de lleno al corazón de esta teoría. Para contestarlas, aunque sea mínimamente y de forma parcial, es conveniente definir el concepto de atractor.

Un conjunto $A$ se denomina atractor global del sistema dinámico (1) si:

- es invariante, es decir si $f(A)=A$ (los puntos de $A$ se transforman en puntos del mismo conjunto), lo que garantiza que las órbitas que comienzan en $A$ permanecen indefinidamente en él y, por tanto, la restricción de $f$ en $A$ es un sistema dinámico sobre $A$;

- es cerrado, esto es, contiene a su frontera, o en otras palabras, las órbitas convergentes $\gamma\left(x_{0}\right)$, con $x_{0} \in A$ (comienzan en puntos de $A$ ), tienen su límite en puntos del propio conjunto $A$;

- y, para todo $x$, se verifica que la distancia de $f(x)$ a $A$ tiende a 0 cuando $t$ tiende a infinito, por lo que todas las órbitas del sistema se acercan al conjunto $A$ ( $A$ atrae a todas las órbitas). Cuando esta propiedad sólo se cumple para $x$ próximos a $A$, se dice que es un atractor local.

4 Especialmente en el capítulo titulado «Valor de la ciencia», que comprende de la página 115 a la 127. 
En consecuencia, la existencia de atractor global sugiere la posibilidad de que la dinámica sobre dicho atractor represente la dinámica a largo plazo del sistema. Típicos ejemplos de atractores son los puntos fijos, las órbitas periódicas y las órbitas cuasiperiódicas (que se suelen asociar, en el caso discreto, con la existencia de curvas cerradas invariantes).

Para ilustrar estos comentarios, consideremos la ecuación (1) y representemos sus órbitas en la recta real. Si las órbitas convergen hacia un punto $x$, que necesariamente cumplirá $f(x)=x$, se obtiene un atractor formado por un punto fijo (véase figura 1.a). Si, por ejemplo, las órbitas se sitúan tras varios instantes en un conjunto formado por cuatro puntos, estaríamos en el caso de un atractor periódico (en la figura 1.b el atractor periódico está formado por los puntos $x_{2}, x_{3}, x_{4}$ y $x_{5}$ ). Otra posibilidad es que las órbitas recorran una curva cerrada simple (que, en la recta real se corresponde con un intervalo cerrado y acotado), lo que indica que no se produce una repetición exacta de los valores y, en general, muestra un comportamiento llamado cuasiperiódico (véase la figura 1.c, en donde la órbita representada rellena completamente el intervalo cerrado $\left[x_{3}, x_{2}\right]$ ).

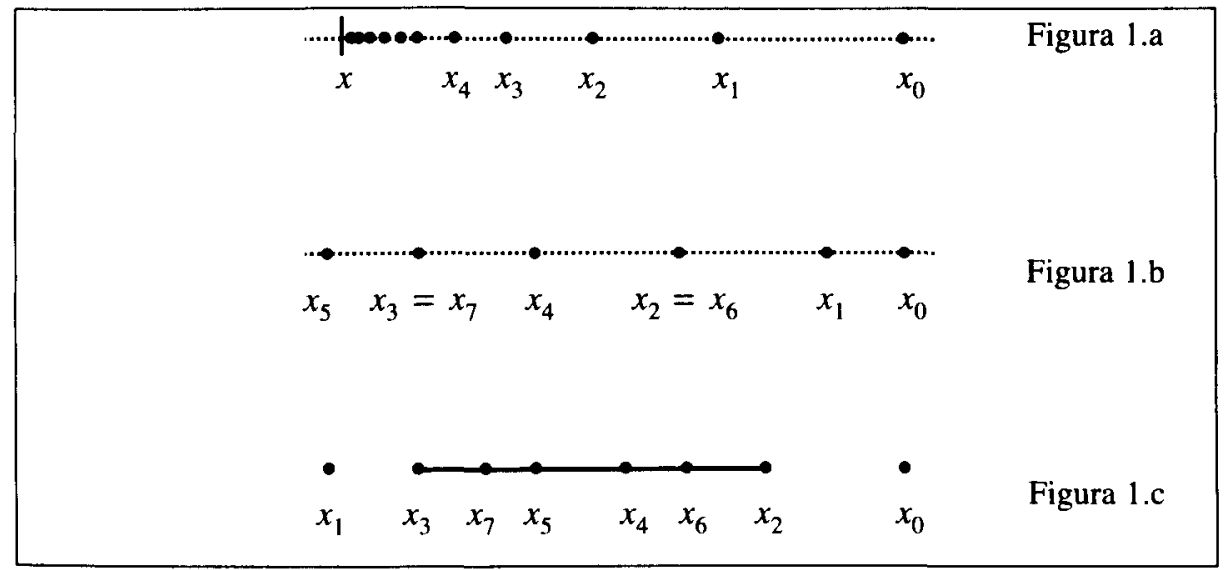

Sin embargo, existen atractores que no tienen forma tan simple (conjuntos finitos, curvas cerradas, o en espacios de mayor dimensión, esferas, toros ${ }^{5}$, etc.), pudiendo ser mucho más irregulares y, de hecho, tener dimensión no entera; estos son los denominados atractores extraños. Naturalmente, el decir que un conjunto tiene dimensión no entera implica la utilización de un determinado concepto de dimensión. Para los propósitos de este trabajo, restringiremos nuestra atención a la más sencilla definición de dimensión de un conjunto: la dimensión de capacidad.

5 Este es el nombre técnico (matemático) de lo que el autor llama, de forma muy ilustrativa aunque un tanto colonialista, donuts. De hecho, podría haber utilizado expresiones como neumático o flotador, que siendo igualmente elocuentes son propias del lenguaje castellano, y mucho más acertadas al ser palabras que hacen referencia a objetos tridimensionales huecos. 
Sea $M(\varepsilon)$ el número mínimo de segmentos de longitud $\varepsilon$ necesarios para recubrir un segmento de longitud $l$; si $\varepsilon$ es pequeño se tiene que $M(\varepsilon)=l \cdot \varepsilon^{l}$. Tomemos ahora un cuadrado de lado $l$ y sea $M(\varepsilon)$ el número mínimo de pequeños cuadrados de lado $\varepsilon$ necesarios para recubrirlo, entonces $M(\varepsilon)=\mathcal{l}^{2} \cdot \varepsilon^{-2}$. Así, en ambos casos $M(\varepsilon)$ se comporta (se escala) como $\varepsilon^{-D}$ cuando $\varepsilon$ tiende a cero, siendo $D$ la dimensión de capacidad; nótese que la dimensión $D$ de cualquier segmento sería 1 y la de cualquier cuadrado 2 (igual que la de cualquier figura en el plano "perfectamente» sólida). Matemáticamente, la dimensión de capacidad de un conjunto $A$ inmerso en un espacio $k$-dimensional viene dada por

$$
D=\lim _{\varepsilon \rightarrow 0} \frac{\operatorname{Ln} M(\varepsilon)}{\operatorname{Ln}(1 / \varepsilon)}
$$

donde $M(\varepsilon)$ es el mínimo número de cubos $k$-dimensionales necesarios para recubrir el conjunto $A$, y a grandes rasgos, trata de medir el grado de ocupación del espacio (cuánto lo rellena) en el que se sitúa el conjunto $A$.

Quizás, el ejemplo más simple de posible atractor extraño es el conjunto de Cantor. Su construcción se ilustra en la figura 2 y se realiza de la siguiente forma: comenzando con el intervalo $[0,1]$, se divide éste en tres partes iguales y se elimina el intervalo abierto central $(1 / 3,2 / 3)$, lo que da como resultado el conjunto unión de $[0,1 / 3]$ y $[2 / 3,1]$; ahora se dividen cada uno de estos dos intervalos en tres partes iguales y de nuevo se eliminan los dos intervalos abiertos centrales, obteniéndose en este paso $[0,1 / 9] \cup[2 / 9,1 / 3]$ $\cup[2 / 3,7 / 9] \cup[8 / 9,1]$; y este proceso se repite indefinidamente.

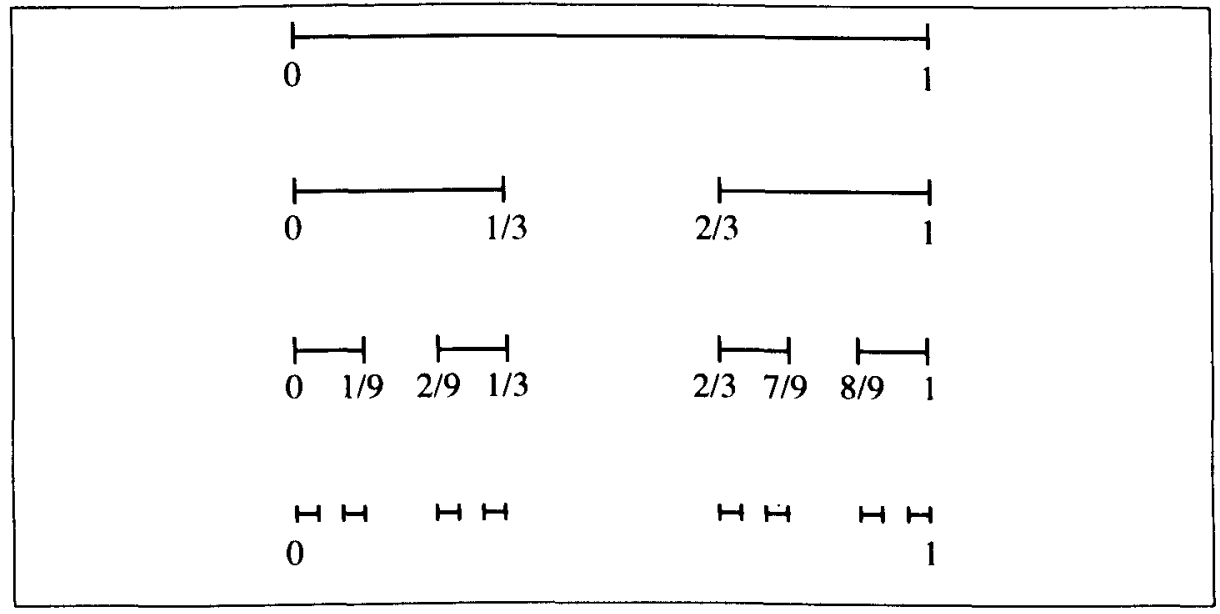

Figura 2

Como se observa en la figura 2, el conjunto de Cantor, que está formado por la unión de infinitos intervalos cerrados disjuntos (con intersección vacía) infinitamente pequeños, tiene dos características un tanto especiales: por un 
lado, autosemejanza, es decir, si se amplia una zona determinada se obtiene una imagen semejante a la de partida y, por otro, dimensión no entera (es posible probar que su dimensión de capacidad es $D=\operatorname{Ln} 2 / \operatorname{Ln} 3 \approx 0.63$ ). Los conjuntos, como sucede con gran número de atractores extraños, que tienen las propiedades de autosemejanza y dimensión no entera se conocen como fractales.

Finalmente, se dice que un sistema exhibe comportamiento caótico si posee un atractor extraño sobre el que la dinámica presenta dependencia sensible a los datos iniciales, lo que significa lo siguiente: órbitas que comienzan muy cercanas (sus datos iniciales son sumamente próximos) se alejan rápidamente (de hecho, exponencialmente). Así, la existencia de caos tiene dos importantes implicaciones. Por un lado, sus órbitas son altamente irregulares, difíciles de distinguir de la órbita de un sistema aleatorio aunque provengan de un sistema determinista. Por otro, pequeños errores crecen rápidamente, alterando por completa la órbita fijada e imposibilitando la predicción a medio y largo plazo.

Por tanto, la dinámica no lineal amplía enormemente el espectro de posibles comportamientos cualitativos de un sistema: si la dinámica lineal es simple y regular, la no lineal puede ser caótica, es decir, compleja y altamente irregular. Sin embargo, y aunque en el texto «Caos y Orden» parece que se establece una identificación entre no linealidad y caos, no conviene olvidar que sistemas no lineales pueden también generar dinámicas simples y regulares, por lo que la característica de no linealidad no es patrimonio exclusivo de los modelos caóticos. Por otro lado, la apariencia de aleatoriedad de las órbitas generadas por un sistema dinámico caótico tampoco debe llevarnos a confusión: son órbitas producidas por un modelo (teórico) determinista, esto es, si se conociese con exactitud el estado inicial se tendría completamente determinada su evolución posterior. Es la incapacidad de una precisión absoluta en la medición empírica del estado inicial ( $y$, de hecho, del estado en cualquier momento) lo que imposibilita la predicción a medio plazo pues, como se ha comentado con anterioridad, estos errores iniciales se propagan agigantándose en cada paso. Naturalmente, a esto se debe añadir que el modelo teórico no deja de ser una aproximación a la realidad, por lo que, aunque puede ser utilizado con fines explicativos de la misma, su capacidad predictiva se ve todavía más reducida.

Muchos de los conceptos que ahora forman parte de la teoría del caos eran ya conocidos en la primera mitad del siglo $\mathrm{XX}$, pero estaban confinados a la comunidad matemática. Al comienzo de la década de los años 70 , y fundamentalmente estimulada por el desarrollo de la informática, la situación cambió rápidamente. El descubrimiento de Lorenz en 1963 de la existencia de un atractor extraño en un modelo teórico de meteorología inició una etapa de posteriores aplicaciones de la teoría del caos en muy diversos contextos: química, mecánica de fluidos, física teórica, ecología, biología, ingeniería, mecánica celeste, economía, etc. Muchos modelos lineales comenzaron a reformularse y, otros que tradicionalmente se habían considerado aleatorios se revisaban desde el prisma de la dinámica compleja; la 
aparente aleatoriedad podía estar generada y, por tanto, explicada por un sistema (determinista) caótico, introduciendo orden en el supuesto desorden. De hecho, la posible existencia de orden (complejo) en sistemas con aspecto anárquico es uno de los pilares sobre los que se sustenta el denominado paradigma del caos.

Estos comentarios permiten de nuevo entender de manera lógica el desarrollo de la Ciencia y subrayan su buen estado de salud: por un lado, los avances teóricos en el campo de la Matemática se convierten en herramienta de otras disciplinas, y los problemas surgidos en estas áreas plantean nuevos retos a los matemáticos, en un proceso de constante retroalimentación; por otro, el descubrimiento de nuevas teorías provoca un replanteamiento profundo de modelos anteriores, en una línea de permanente progreso, no con la intención de olvidarlos (y mucho menos repudiarlos), sino más bien con el sano propósito de perfeccionarlos y así contribuir a mejorar la explicación de la realidad. En este sentido conviene notar que, si bien muchas de las teorías actuales refutan (total o parcialmente) otras anteriores, la mayor parte de ellas serán, muy posiblemente, también negadas y/o perfeccionadas en siglos venideros.

La teoría de sistemas dinámicos también proporciona una metodología de análisis de modelos cuya estructura depende de factores que pueden cambiar en ciertos momentos. Esto permite explicar situaciones en las que el atractor, y por tanto el comportamiento cualitativo del sistema, sufre alteraciones, incluso profundas transformaciones. Para ilustrar este punto basta considerar el sistema

$$
x_{t}=f\left(\mu, x_{t-1}\right)
$$

donde $\mu$ es un parámetro en principio fijo. Supongamos que para un valor inicial $\mu=\mu_{0}$ el sistema tiene un atractor $A_{0}$. Si el parámetro varía su valor (digamos ahora pasa a ser $\mu=\mu_{1}$ ), el sistema y el atractor también cambian (denotemos al nuevo atractor por $A_{l}$ ). Si las características cualitativas de $A_{0}$ y $A_{l}$ son estructuralmente similares, el comportamiento cualitativo del sistema no sufre grandes cambios (piénsese por ejemplo que $A_{0}$ es una circunferencia y $A_{l}$ una elipse). Sin embargo, cabe la posibilidad de que el nuevo atractor sea muy distinto del inicial, incluso pasar de un atractor simple a uno complejo. En este caso, se dice que el sistema presenta en algún punto $\mu_{c}$. intermedio entre $\mu_{0}$ y $\mu_{1}$, una bifurcación.

Sin entrar en más detalle técnico, los sistemas dinámicos parametrizados permiten analizar fenómenos cuyo comportamiento dinámico cualitativo cambia substancialmente con el transcurso del tiempo y, con frecuencia, suelen estar asociados con procesos autoorganizativos. Desde este punto de vista, cambios en el sistema se transfieren a cambios en el comportamiento dinámico del mismo, y estos hacen de nuevo cambiar el propio sistema. El nivel de organización en cada instante vendría determinado por el tipo de atractor, pudiendo el sistema pasar de organizaciones muy simples a otras extremadamente complejas, o viceversa. En este contexto, la organización compleja se 
corresponde con la existencia de atractor caótico (proveniente de un modelo determinista, no aleatorio), con orden intrínseco. Así, en toda esta construcción teórica se supone implícitamente la existencia de una ley de cambio, por lo que, cuanto menos, es discutible su aplicación a fenómenos históricos, políticos o sociales.

Con esta panorámica de los sistemas dinámicos, consideramos que algunas de las conclusiones de la obra no parecen tan evidentes. Si tomamos como ejemplo la aplicación de la dinámica caótica a la Economía, comprobamos como, mediante la modelización teórica de interacciones no lineales entre las variables, se ha conseguido explicar cambios en los ciclos de actividad, cambios que pueden ser perfectamente irregulares y desordenados. Es decir, el sistema ha generado un comportamiento complejo susceptible de explicar cambios de este tipo en la realidad. En este sistema las interacciones entre agentes económicos están determinadas por el modelo teórico, de modo que los agentes no poseen la capacidad de actuar diferente, no poseen libertad. Extrapolar este paradigma también a la Historia o a la Política nos conduce a reducir la conducta humana a una regla no lineal, capaz de generar en el sistema un comportamiento agregado irregular. Por tanto, queda en entredicho que la adopción de este paradigma haga concebir al individuo como naturaleza, pleno de espontaneidad.

A pesar de todo esto, bien podríamos profesar que este tipo de fenómenos (históricos, políticos y sociales en general) estuvieran determinados por un sistema dinámico con comportamiento caótico, por complejo que fuera y pese a nuestro desconocimiento del mismo. Si esto fuera así, plantearía un panorama ciertamente idílico para la ciencia actual; ahora únicamente restaría descubrir los modelos teóricos que rigen y gobiernan los procesos considerados. La Historia, por otro lado, nos indica que la teoría del caos no es el fin de la ciencia, no es el último escalón científico, sino que, con toda seguridad, esta teoría es un paso más para la comprensión y entendimiento de ciertos procesos causales, y que en modo alguno mediante ella seremos capaces de explicar toda la realidad.

Es cierto que esta teoría se presenta en un marco muy específico (en el contexto de los sistemas dinámicos) que posee la ventaja de ser abstracto, lo que le da una mayor universalidad potencial. En este punto radica el enorme impacto que ha causado, aplicándose en muy diferentes campos, hablándose de un paradigma unificador de la ciencia e incluso proponiéndose el nacimiento de una nueva ciencia, la Ciencia del Caos (véase Gleick, 1988). Sin embargo, esto no significa ni que todo fenómeno pueda ser explicado por un modelo caótico ni que la teoría del caos pueda ser aplicada a cualquier situación. Antes lo contrario, aunque la aportación, como hemos comentado, a la Economía Teórica está siendo satisfactoria, inyectando incluso dosis de historia en la dinámica económica -bifurcaciones y dependencia del camino seguido (path-dependence)-, la aplicación al dominio político-histórico, a nuestro juicio, se queda en un punto de vista retórico y metafórico. 


\section{REFERENCIAS}

Devaney, R. L., 1989. An introduction to chaotic dynamical systems. 2." edición. Addison-Wesley.

Gleick, J., 1988. Caos. La creación de una ciencia. Seix Barral.

KIM, J. H. y Stringer, J., eds., 1992. Applied chaos. Wiley-Interscience.

LoRenz, E. N., 1995. La esencia del caos. Editorial Debate.

Nicolis, G. y Prigogine, I., 1994. La estructura de lo complejo: en el camino hacia una nueva comprensión de las ciencias. Alianza Universidad.

OTT, E., 1993. Chaos in dynamical systems. Cambridge University Press.

Prigogine, I., 1997. Las leyes del caos. Editorial Crítica. 